\title{
Universal graph description for one-dimensional exchange models
}

\author{
Jean Decamp $\odot,{ }^{1,2,3}$ Jiangbin Gong, ${ }^{3}$ Huanqian Loh $\odot,{ }^{2,3}$ and Christian Miniatura $\odot^{1,2,3,4,5,6}$ \\ ${ }^{1}$ MajuLab, Centre National de la Recherche Scientifique-UCA-SU-NUS-NTU International Joint Research Unit, Singapore, Singapore \\ ${ }^{2}$ Centre for Quantum Technologies, National University of Singapore, 117543 Singapore, Singapore \\ ${ }^{3}$ Department of Physics, National University of Singapore, Singapore 117542, Singapore \\ ${ }^{4}$ School of Physical and Mathematical Sciences, Nanyang Technological University, 637371 Singapore, Singapore \\ ${ }^{5}$ Yale-NUS College, Singapore 138527, Singapore \\ ${ }^{6}$ Université Côte d'Azur, Centre National de la Recherche Scientifique, INPHYNI, Nice, France
}

(Received 3 June 2020; revised 4 August 2020; accepted 5 August 2020; published 24 August 2020)

\begin{abstract}
We demonstrate that a large class of one-dimensional quantum and classical exchange models can be described by the same type of graphs, namely, Cayley graphs of the permutation group. Their well-studied spectral properties allow us to derive crucial information about those models of fundamental importance in both classical and quantum physics, and to completely characterize their algebraic structure. Notably, we prove that the spectral gap can be obtained in polynomial computational time, which has strong implications in the context of adiabatic quantum computing with quantum spin chains. This quantity also characterizes the rate to stationarity of some important classical random processes such as interchange and exclusion processes. Reciprocally, we use results derived from the celebrated Bethe ansatz to obtain mathematical results about these graphs in the unweighted case. We also discuss extensions of this unifying framework to other systems, such as asymmetric exclusion processes-a paradigmatic model in nonequilibrium physics—or the more exotic non-Hermitian quantum systems.
\end{abstract}

DOI: 10.1103/PhysRevResearch.2.033297

\section{INTRODUCTION}

Consider the following situations: (a) a card shuffling, where at each step two randomly chosen adjacent cards of the deck are being switched; (b) a cold atom experiment involving strongly interacting ${ }^{173} \mathrm{Yb}$ atoms confined in one dimension; (c) the quantum Heisenberg $X X X$ spin chain; and (d) the protein synthesis on RNA. What do these situations have in common? They can all be described by one-dimensional exchange models, where the action of the Hamiltonian in the quantum case or of the transition matrix in the classical stochastic case is to exchange two adjacent elements [1-5]. In fact, one of the purposes of this paper is to show that they are all described by the same theoretical object, namely, a graph associated with the permutation group.

One-dimensional (1D) exchange models are ubiquitous in both quantum and classical physics, and their study has been associated with important theoretical breakthroughs. It was in particular to solve the homogeneous 1D Heisenberg spin chain, a model of fundamental importance for the study of quantum magnetism [6], that Bethe introduced his celebrated ansatz in 1931 [7]. His powerful insight, which attracted little attention at first, turned out to be one of the most fruitful

Published by the American Physical Society under the terms of the Creative Commons Attribution 4.0 International license. Further distribution of this work must maintain attribution to the author(s) and the published article's title, journal citation, and DOI. theoretical achievements of the last century, as it has been extended and applied to a wide range of quantum [8-12] and classical [13-15] models that are said to be quantum integrable [16]. However, extracting any physically relevant information from this method is still strenuous, and, more importantly, the Bethe ansatz can no longer be applied if the system is inhomogeneous, as one could expect in a realistic experimental situation [17]. In this case, the study of such strongly correlated systems is greatly challenged by their computational complexity.

In this paper, we develop a universal graph-theoretical description in order to understand 1D exchange models containing inhomogeneities, with both periodic and open boundary conditions (respectively, $\mathrm{PBC}$ and $\mathrm{OBC}$ ). For its central importance, a peculiar focus is given to the inhomogeneous Heisenberg spin chain and its generalization to $S U(\kappa)$ exchange spin chains [18]. This powerful framework allows us to derive crucial results about the system, which are true for any system size, number of spin orientations, and spin configurations.

(1) We completely identify the algebraic structure of the system (i.e., its decomposition according to the irreducible representation of the permutation group). The following results are consequences of this fundamental decomposition.

(2) We demonstrate a generalized Lieb-Mattis theorem [19] by identifying how the energy levels are ordered as a function of their symmetries in both the ferromagnetic and antiferromagnetic cases.

(3) In the ferromagnetic case, we show how the energy gap between the ground state and first excited state can 
be obtained in polynomial computational time. This huge computational advantage has important consequences in the field of adiabatic quantum computing, where the speed of the process is limited by the value of the energy gap-which is a priori an exponentially hard quantity to compute in the strongly correlated systems involved [20].

The second important claim of this paper is that the integrable case where the Bethe ansatz may be applied corresponds, within our graph-theoretical framework, to the peculiar case where the graphs are unweighted. As a consequence, we show that results derived from the Bethe ansatz may be applied in order to study the spectra of Cayley graphs of the permutation group with large number of elements. This approach should attract the attention of mathematicians.

Furthermore, we discuss how our graph-theoretical framework and its generalizations may be applied to study a large class of quantum and classical 1D exchange models. These include the Fermi-Hubbard model and its non-Hermitian generalization for the quantum part, as well as 1D stochastic processes for the classical part, such as the interchange process and symmetric and asymmetric exclusion processes. The latter are paradigmatic in the context of nonequilibrium statistical physics [15].

Some of the groundwork for our results was first laid in Ref. [21] for a peculiar type of exchange model, namely, strongly repulsive mixtures of fermions confined in inhomogeneous continuous 1D potentials with OBC. As further explained in the main text, the results we present here are significantly stronger and universal.

\section{GRAPH-THEORETICAL DESCRIPTION OF INHOMOGENEOUS $S U(\kappa)$ EXCHANGE SPIN CHAINS}

\section{A. An exchange model}

We consider a system of $N$ spin $S, S$ being a half integer, on a $1 \mathrm{D}$ closed $(\mathrm{PBC})$ or open (OBC) chain. We suppose that the populations in each spin component are fixed and given by a partition $v=\left(N_{1}, \ldots, N_{\kappa}\right)$ of $N$, i.e., such that $N_{1} \geqslant$ $\cdots \geqslant N_{\kappa}$ and $N_{1}+\cdots+N_{\kappa}=N$, with $\kappa \in\{2, \ldots, 2 S+1\}$. The Hamiltonian of the model is

$$
\mathcal{H}=\sum_{k=1}^{\tilde{N}} J_{k}\left(\mathbb{1}-P_{k, k+1}\right),
$$

where the local interaction constants $J_{k}$ verify $J_{k}>0\left(J_{k}<0\right)$ for all $k$ in the ferromagnetic (antiferromagnetic) case, and operator $P_{k, k+1}$ exchanges the spin orientations at positions $k$ and $k+1$, e.g.,

$$
P_{3,4}|\uparrow \uparrow \downarrow \uparrow \downarrow \uparrow\rangle=|\uparrow \uparrow \uparrow \downarrow \downarrow \uparrow\rangle .
$$

We have used the convention $\tilde{N}=N$ and $N+1=1$ for PBC and $\tilde{N}=N-1$ for OBC. Equivalently, we could have studied the energy-shifted Hamiltonian

$$
\tilde{\mathcal{H}}=-\sum_{k=1}^{\tilde{N}} J_{k} P_{k, k+1},
$$

but our graph-theoretical description will be more straightforward using Eq. (1). A complete study of the Hamiltonian in
Eq. (3) for the homogeneous case $J_{1}=\cdots=J_{\tilde{N}}=1$ using the Bethe ansatz was conducted by Sutherland [18].

As first observed by Dirac [22], in the case where $S=1 / 2$, $\mathcal{H}$ is equivalent to the Heisenberg spin chain. Indeed, one can write $P_{k, k+1}$ as

$$
\begin{aligned}
P_{k, k+1} & =\frac{\mathbb{1}+\sigma_{k}^{z} \sigma_{k+1}^{z}}{2}+\sigma_{k}^{+} \sigma_{k+1}^{-}+\sigma_{k}^{-} \sigma_{k+1}^{+} \\
& =\frac{1}{2}\left(\mathbb{1}+\vec{\sigma}_{k} \cdot \vec{\sigma}_{k+1}\right),
\end{aligned}
$$

where we have used the standard notations for the Pauli matrices $\vec{\sigma}=\left(\sigma^{x}, \sigma^{y}, \sigma^{z}\right)$ and the raising and lowering operators $\sigma^{ \pm}$acting at positions $k$ and $k+1$. Therefore, the Heisenberg Hamiltonian of the $X X X$ spin chain verifies

$$
\mathcal{H}_{X X X}=-\sum_{k=1}^{\tilde{N}} J_{k} \vec{\sigma}_{k} \cdot \vec{\sigma}_{k+1}=2 \mathcal{H}-\sum_{k=1}^{\tilde{N}} J_{k} \mathbb{1} .
$$

In other words, the Heisenberg model is, up to an affine transformation, an exchange model. More generally, for spins larger than $1 / 2, P_{k, k+1}$ can always be written (up to an affine transformation) as a scalar product $\vec{\gamma}_{k} \cdot \vec{\gamma}_{k+1}$, where $\vec{\gamma}$ is the vector of all the generators $\left(\gamma^{i}\right)_{1 \leqslant i \leqslant \kappa^{2}-1}$ of the $S U(\kappa)$ algebra [23]. For instance, in the $S U(\kappa=3)$ case, one has

$$
P_{k, k+1}=\frac{1}{3} \mathbb{1}+\frac{1}{2} \vec{\lambda}_{k} \cdot \vec{\lambda}_{k, k+1},
$$

where $\vec{\lambda}$ is composed of the eight Gell-Mann matrices. Note that one also has $P_{k, k+1}=\vec{S}_{k} \cdot \vec{S}_{k+1}+\left(\vec{S}_{k} \cdot \vec{S}_{k+1}\right)^{2}-1$ with $\vec{S}=\left(S_{x}, S_{y}, S_{z}\right)$ the three-dimensional spin-1 operator [18]. Thus, $\mathcal{H}$ may be seen as a $S U(\kappa)$ generalization of the Heisenberg spin chain.

For a given mixture $v=\left(N_{1}, \ldots, N_{\kappa}\right)$, the number of distinguishable spin configurations along the chain is given by the following multinomial coefficient:

$$
D_{\nu}=\frac{N !}{N_{1} ! \cdots N_{\kappa} !} .
$$

Writing as $|\chi\rangle$ the quantum state associated with a given spin configuration $\chi$, each eigenvector $|\psi\rangle$ of $\mathcal{H}$ can be written $|\psi\rangle=\sum_{\chi} a_{\chi}|\chi\rangle$, with $\vec{a}=\left(a_{\chi}\right)_{\chi}$ a vector of length $D_{v}$. Within this representation, $\mathcal{H}$ is a $D_{v} \times D_{v}$ real symmetric matrix $\Delta^{v}$ defined as

$$
\Delta_{\chi \chi^{\prime}}^{v}=\left\{\begin{array}{ll}
-J_{k} & \text { if }|\chi\rangle=P_{k, k+1}\left|\chi^{\prime}\right\rangle \neq\left|\chi^{\prime}\right\rangle \\
\sum_{k \in K_{\chi}} J_{k} & \text { if }|\chi\rangle=\left|\chi^{\prime}\right\rangle \\
0 & \text { otherwise }
\end{array},\right.
$$

where $K_{\chi}=\left\{k: P_{k, k+1}|\chi\rangle \neq|\chi\rangle\right\}$.

As it is typically the case for strongly correlated systems, the dimension of the problem grows exponentially with the size $N$ of the system, and brute-force diagonalization becomes intractable even for moderate values of $N$. A comprehensive framework is thus necessary in order to overcome complexity and make general conclusions.

\section{B. Interpretation in terms of graph theory}

We suppose that the reader is familiar with basic notions of graph and group theories. Relevant definitions and properties as well as common references are included in the Appendices. 
We recall here the definitions of Cayley and Schreier graphs [24]. Let $G$ be a finite group, and let $S$ be a generating subset of $G$ [25] such that $s \in S$ if and only if $s^{-1} \in S$ ( $S$ is said to be symmetric). The Cayley graph $X(G, S)$ associated with $G$ and $S$ is the graph the vertices of which are indexed by all the elements of $G$, and such that there is an edge between $g$ and $g^{\prime}$ if there is $s \in S$ such that $g^{\prime}=s g$. The fact that $S$ is generating and symmetric ensures that $X(G, S)$ is connected and undirected (respectively). Additionally, if we consider a subgroup $H$ of $G$, the Schreier graph $X(H \subset G, S)$ is defined by indexing the vertices by all the cosets $g H$, and such that there is an edge between $g H$ and $g^{\prime} H$ if there is $s \in S$ such that $g^{\prime} H=s g H \neq g H$. The fact that $g^{\prime} H \neq g H$ ensures that there are no self-loops. Note that if $H$ is the trivial subgroup $X(H \subset G, S) \cong X(G, S)$. Additionally, one could have easily defined the weighted versions of $X(G, S)$ and $X(H \subset G, S)$ by associating each $s \in S$ to a weight $w_{s} \in \mathbb{R}$.

Then, our graph-theory interpretation of Hamiltonian $\mathcal{H}$ and of its representation $\Delta^{v}$ is the following: $\Delta^{v}$ is the Laplacian matrix of the weighted Schreier graph $X\left(\mathfrak{S}_{v} \subset \mathfrak{S}_{N}, \tilde{S}_{C}\right)$, where we have the following.

(1) $\mathfrak{S}_{N}$ is the permutation group of $\{1, \ldots, N\}$.

(2) $\mathfrak{S}_{v}=\mathfrak{S}_{N_{1}}^{v} \times \cdots \times \mathfrak{S}_{N_{K}}^{v}$ is the Young subgroup associated with $v$, with $\mathfrak{S}_{N_{i}}^{v}$ the set of permutations $P \in \mathfrak{S}_{N}$ such that $P(i)=i$ if $i$ does not belong to $\left\{N_{1}+\cdots+N_{i-1}+\right.$ $\left.1, \ldots, N_{1}+\cdots+N_{i-1}+N_{i}\right\}$.

(3) $\tilde{S}_{C} \equiv\{(1,2),(2,3), \ldots,(\tilde{N}, \tilde{N}+1)\}$ is the set of nearest-neighbor transpositions (Coxeter generators), where each $(k, k+1) \in \tilde{S}_{C}$ is associated with a weight $J_{k}$.

Note that the only difference between OBC and PBC is the presence of transposition $(N, 1)$ in the PBC case. As a pedagogical example, the graphs associated with a $(2,2)$ mixture of two spin-up and two spin-down with both OBC and $\mathrm{PBC}$ are displayed in Fig. 1. Other examples of graphs for more complex spin mixtures with PBC are given in Fig. 2.

\section{Results}

A first trivial observation is that, as it is well known for Laplacian matrices, the spectrum $\sigma\left(\Delta^{v}\right)$ of $\Delta^{v}$ is given by real positive numbers and contains zero with multiplicity 1 . Indeed, the multiplicity of zero in the spectrum of the Laplacian matrix is equal to the number of connected components of the graph, and here $X\left(\mathfrak{S}_{v} \subset \mathfrak{S}_{N}, \tilde{S}_{C}\right)$ is connected since $\tilde{S}_{C}$ generates $\mathfrak{S}_{N}$ (see Appendix A). Therefore, the ground state of $\mathcal{H}$ in the ferromagnetic case is nondegenerate with zero energy.

\section{Algebraic structure}

One great advantage of noting that a graph is a Cayley or Schreier graph is that it allows one to access the algebraic structure of its spectrum in a natural way. Indeed, by associating a matrix to a graph that is itself associated with a group, we associate a matrix to a group: In other words, we are using the well-studied language of linear representations [26-28]. In our case, for a given spin mixture $v$, we observe that each spin configuration is equivalent to a unique tabloid of shape $v$ [29]. The identification is done as follows. First, we associate each spin configuration $|\chi\rangle$ to a tabloid of shape $v$. Then, we associate each row $i \in\{1, \ldots, \kappa\}$ to one spin orientation, and

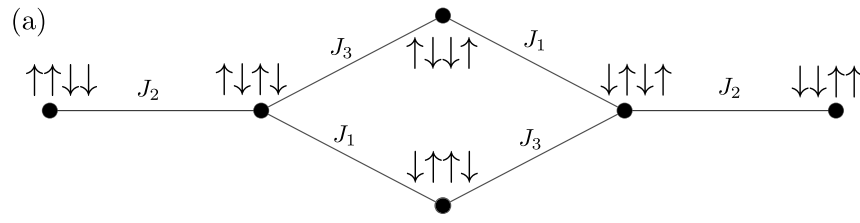

(b)

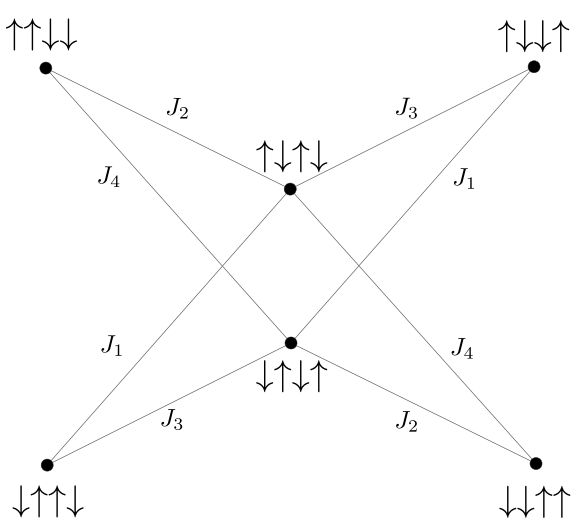

FIG. 1. Graphs describing a Heisenberg spin chain $(2,2)$ of two spin-up and two spin-down. (a) Open boundary conditions (OBC). (b) Periodic boundary conditions (PBC). The graph with OBC can be obtained from the graph with $\mathrm{PBC}$ by removing the edges with weight $J_{4}$, corresponding to exchanges between distinguishable spins in positions 1 and 4 . One can easily check that the Laplacian matrices of these graphs are equal to $\Delta^{(2,2)}$ [Eq. (8)].

we identify each index of row $i$ as the position of a spin of type $i$ along the chain. For instance, consider a mixture $(5,3)$ of five spin-up and three spin-down. Then, the following spin configuration and tabloid are equivalent:

$$
|\uparrow \downarrow \uparrow \uparrow \uparrow \downarrow \uparrow \downarrow\rangle \Leftrightarrow \begin{array}{lllll}
\hline 1 & 3 & 4 & 5 & 7 \\
\hline 2 & 6 & 8 & &
\end{array} .
$$

Therefore, $\Delta^{v}$ is written in the vector space the basis of which is indexed by the tabloids, namely, the permutation module $M^{v}$. More precisely, it can be written as

$$
\begin{aligned}
\Delta^{v} & =\sum_{k=1}^{\tilde{N}} J_{k}\left\{M^{v}(\mathrm{Id})-M^{v}[(k, k+1)]\right\} \\
& =M^{v}\left\{\sum_{k=1}^{\tilde{N}} J_{k}[\mathrm{Id}-(k, k+1)]\right\},
\end{aligned}
$$

where we have linearly extended $M^{v}$ to an algebra representation and Id and $(k, k+1)$ are, respectively, the identity and transposition in positions $k, k+1$ of $\mathfrak{S}_{N}$. Note that we just have rewritten Eq. (1) in terms of representation theory. The interest of such a rewriting is that the decomposition of $M^{v}$ according to the irreducible representations (irreps) $S^{\mu}$ of $\mathfrak{S}_{N}$ (where the partition $\mu$ of $N$ labels uniquely the irrep) is well 
(a)

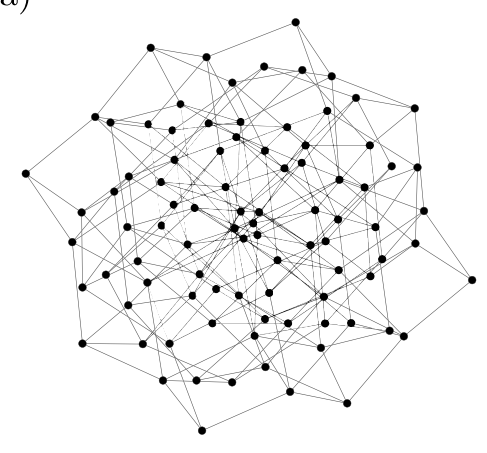

(b)

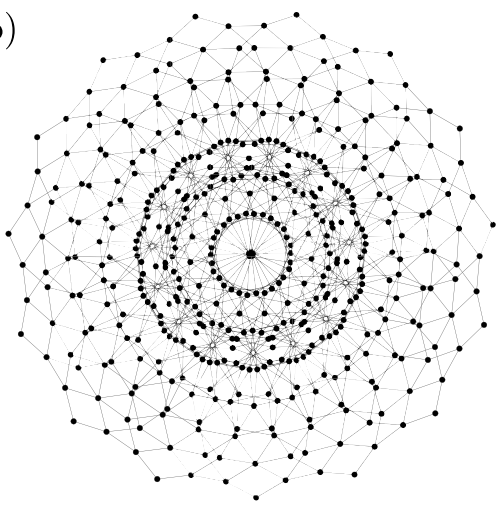

(c)

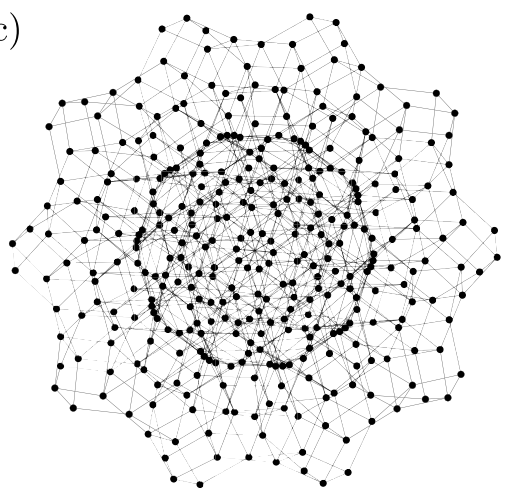

FIG. 2. Examples of graphs corresponding to different spin mixtures on closed chains (PBC): (a) $(2,2,2),(b)(12,3)$, and (c) (7,2,1). These graphs display remarkable symmetry properties, with a characteristic rose shape that is reminiscent of the boundary conditions.

known and given by the so-called Young's rule [30]:

$$
M^{v} \cong \bigoplus_{\mu \unrhd v} k_{\mu \nu} S^{\mu} \quad\left(k_{\nu \nu}=1\right)
$$

Here, the multiplicities $k_{\mu \nu}$ are positive integers known as the Kostka numbers, and $\unrhd$ is the dominance order over the set of partitions of $N$, which states that $\left[\mu_{1}, \ldots, \mu_{r}\right] \unrhd\left[v_{1}, \ldots, v_{r}\right]$ if $\mu_{1}+\cdots+\mu_{k} \geqslant v_{1}+\cdots+v_{k}$ for all $k$. Thus, we obtain

$$
\Delta^{\nu} \cong \bigoplus_{\mu \unrhd v} k_{\mu \nu} S^{\mu}\left\{\sum_{k=1}^{\tilde{N}} J_{k}[\operatorname{Id}-(k, k+1)]\right\},
$$

with $k_{v v}=1$. Equation (12) is a central result. Translated into words, it means that $\Delta^{\nu}$ is block diagonal, and contains $k_{\mu \nu}$ identical blocks of size $\operatorname{dim} S^{\mu}$ corresponding to the irreps $S^{\mu}$ where $\mu \unrhd v$. The fact that it does not contain irreps $S^{\mu^{\prime}}$ such that $\nu \triangleright \mu^{\prime}$ can be interpreted is a consequence of the Pauli principle.

\section{Symmetry ordering} $v^{\prime}$

Equation (12) implies that, given two spin mixtures $v$ and

$$
v \unrhd v^{\prime} \Rightarrow \sigma\left(\Delta^{v}\right) \subseteq \sigma\left(\Delta^{v^{\prime}}\right) .
$$

In particular, the spectral radii (the largest absolute value of the eigenvalues) verify $\rho\left(\Delta^{v}\right) \leqslant \rho\left(\Delta^{v^{\prime}}\right)$. Importantly, one can then show the important fact that it is always possible to construct an eigenvector $\vec{a}_{v}$ with eigenvalue $\rho\left(\Delta^{v}\right)$ that belongs to the symmetry class $[v]$ (in other words, which is "as antisymmetric as possible"). The proof of this statement is exactly the same as the one we described in Ref. [21]: First we note that the graph $X\left(\mathfrak{S}_{v} \subset \mathfrak{S}_{N}, \tilde{S}_{C}\right)$ is bipartite and can be separated into even and odd spin configurations $|\chi\rangle$, then we use this fact to construct a vector that belongs to the symmetry class $[v]$ and show that it belongs to the eigenspace with eigenvalue $\rho\left(\Delta^{v}\right)$. Note that the fact that $k_{v v}=1$ in Eq. (12) implies that $\vec{a}_{v}$ is in fact unique for generic choices of weights $\left(J_{k}\right)_{k}$ (see also Ref. [31]). In the context of Ref. [21], the fact that the spectrum of $\Delta^{v}$ has a negative contribution to the energy implies that $\rho\left(\Delta^{v}\right)$ corresponds to the ground state of a given mixture $v$. Therefore, the fact that this ground state belongs to the symmetry class $[v]$ has been interpreted as a generalization of the Lieb-Mattis theorem [19]. Here, the ground-state energy is $-\rho\left(\Delta^{v}\right)$ for the antiferromagnetic case $J_{k}<0$, and we can reach a conclusion similar to Ref. [21]. Nevertheless, the situation is different for the ferromagnetic case $J_{k}>0$, where $\rho\left(\Delta^{v}\right)$ corresponds to the maximal energy of the mixture $v$, which is of course less physically interesting. It is, however, an important lemma for what follows. Indeed, Eq. (13) also shows that all the spectra are included in the spectrum of $\Delta^{(1, \ldots, 1)} \equiv \Delta$ corresponding to the case where $N=\kappa$. In this case, $X\left(\mathfrak{S}_{(1, \ldots, 1)} \subset \mathfrak{S}_{N}, \widetilde{S}_{C}\right)$ is isomorphic to the Cayley graph $X\left(\mathfrak{S}_{N}, \tilde{S}_{C}\right)$. It is a bipartite graph, since it can be split between even and odd permutations. It is also $d$ regular, with

$$
d=\sum_{k=1}^{\tilde{N}} J_{k}
$$

Therefore, one has

$$
\lambda \in \sigma(\Delta) \quad \Leftrightarrow \quad 2 d-\lambda \in \sigma(\Delta) .
$$

Indeed, if $\vec{a}_{\lambda}$ is an eigenvector of $\Delta$ with eigenvalue $\lambda$, it is easy to see that the vector $\tilde{a}_{\lambda}$ that coincides with $\vec{a}_{\lambda}$ for vertices corresponding to even permutations and is equal to $-\vec{a}_{\lambda}$ for odd vertices is an eigenvector of $\Delta$ with eigenvalue $2 d-\lambda$. Moreover, if $\vec{a}_{\lambda}$ belongs to the symmetry class [ $\mu$ ], we observe that, by construction, $\tilde{a}_{\lambda}$ belongs to the conjugate symmetry class $\left[{ }^{t} \mu\right]$. For instance, $0 \in \Delta$ belongs to the trivial representation $[N]$ (totally symmetric) and $2 d=\rho(\Delta)$ belongs to the sign representation $[1, \ldots, 1]$ (totally antisymmetric). More generally, if we denote by $E([\mu])$ the lowest eigenvalue of $\Delta$ that belongs to the symmetry class $[\mu]$, we deduce from the previous discussion that, with both $\mathrm{OBC}$ and $\mathrm{PBC}$, the following generalized Lieb-Mattis theorem holds:

$$
\mu \unrhd \mu^{\prime} \Rightarrow\left\{\begin{array}{ll}
E([\mu]) \leqslant E\left(\left[\mu^{\prime}\right]\right) & \text { if } J_{k}>0 \\
E([\mu]) \geqslant E\left(\left[\mu^{\prime}\right]\right) & \text { if } J_{k}<0
\end{array} .\right.
$$

Thus, we observe opposite behavior in the ferromagnetic and antiferromagnetic phases, where the ground state tends to be more symmetric in the first case and more antisymmetric in the second case. 


\section{Energy gap}

Let us discuss the ferromagnetic case, as the antiferromagnetic is similar to what we described in Ref. [21]. If we denote the spectral gap of $\Delta$ by $\lambda_{*}$, which in this case is the smallest nonzero eigenvalue, the fact that $\operatorname{dim} S^{[N]}=1$, together with the fact that zero has multiplicity of 1 and that $[N-1,1] \unrhd \mu$ for any partition $\mu \neq[N]$, implies

$$
\lambda_{*}=E([N-1,1]) .
$$

Additionally, it is easy to deduce from Eq. (12) that $\lambda_{*}$ is in fact the spectral gap of $\Delta^{v}$ for any $v \neq(N)$. In other words, for any spin mixture, the energy gap is the same as in the polaron case with $N-1$ spin-up and one spin-down. It is therefore sufficient to compute the lowest nonzero eigenvalue of the following $N \times N$ matrix:

$$
\left(\begin{array}{ccccc}
J_{1}+J_{N} & -J_{1} & & & -J_{N} \\
-J_{1} & J_{1}+J_{2} & -J_{2} & & \\
& \ddots & \ddots & \ddots & \\
& & -J_{N-2} & J_{N-1}+J_{N-2} & -J_{N-1} \\
-J_{N} & & & -J_{N-1} & J_{N-1}+J_{N}
\end{array}\right),
$$

where $J_{N}=0$ in the OBC case. Depending on the boundary conditions, Eq. (18) is either the Laplacian matrix $\Delta^{(N-1,1)}$ of a cycle graph $C_{N}(\mathrm{PBC}$, here, e.g., for $N=9)$,

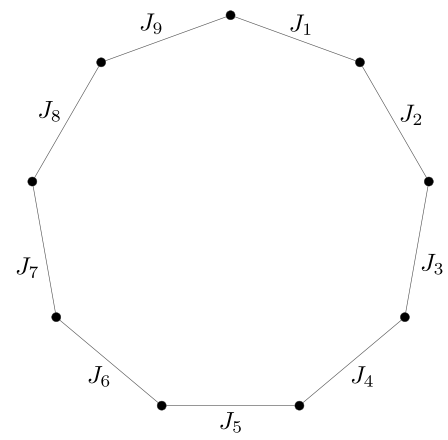

or a path graph $P_{N}(\mathrm{OBC}$-here, e.g., for $N=9)$ :

- $J_{1}$. $J_{2}, J_{3}, J_{4}, J_{5}, J_{6}, J_{7}, J_{8}$.

This considerably reduces the complexity of the problem (typically from $N$ ! to $N$ ). Note that Bacher has given an alternative proof of Eq. (17) for the unweighted Cayley graph $X\left(\mathfrak{S}_{N}, S_{C}\right)$ with $S_{C}=\{(1,2),(2,3), \ldots,(N-1, N)\}$ in a purely mathematical context [32]. Here, our result relies only on symmetry arguments. We also remark that in Ref. [21] the fact that the Laplacian spectrum has a negative contribution to the energy implies that one can only use Eq. (17) in the case $N=\kappa$ where Eq. (15) is valid. Here, in the ferromagnetic case, the result is much stronger, since one can use this method for any spin mixture $v$. This result is illustrated in Fig. 3.

Thus, the application of these results to adiabatic quantum computing, where the speed of the process depends crucially on the value of the energy gap, is extremely promising [20]. For instance, with 100 qubits with 50 spin-up and 50 spindown that are subjected to $\mathcal{H}$, one only has to diagonalize the $\Delta^{(99,1)}$ matrix of size $100 \times 100$ in order to get the spectral gap, instead of the whole $\Delta^{(50,50)}$ matrix of size $D_{(50,50)} \times$ $D_{(50,50)} \approx 10^{29} \times 10^{29}$. A subsequent question for future work

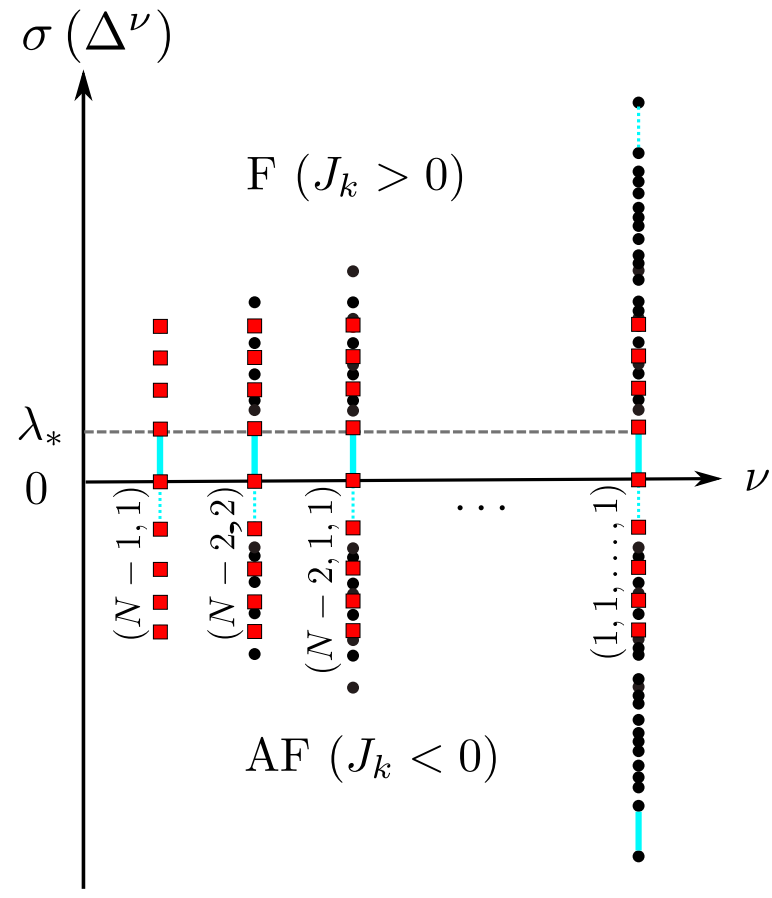

FIG. 3. Spectra $\sigma\left(\Delta^{v}\right)$ of the Laplacian matrices $\Delta^{v}$ for different spin mixtures $v$, where $v$ is a partition of $N$, in both the ferromagnetic $\left(F, J_{k}>0\right)$ and antiferromagnetic $\left(A F, J_{k}<0\right)$ cases. In the $F$ phase, the energy gap $\lambda_{*}$ of the simplest nontrivial case $(N-1,1)$ is the same as the energy gap of any mixture $v$, which gives a huge computational advantage. This is no longer true in the $A F$ phase, where the spectra are opposite as compared to the $F$ phase, so that $\lambda_{*}$ is associated with the energy differences between the most and second-most excited states. The only exception is in the case where $v=(1,1, \ldots, 1)$ (i.e., $N=\kappa)$, due to the symmetry of the spectrum in this case [Eq. (15)]. $\lambda_{*}$ is represented by a solid (dashed) light blue line when associated with the energy gap (the gap between the second-most and most excited states). The spectrum of $\Delta^{(N-1,1)}$, which is included in $\sigma\left(\Delta^{v}\right)$ for all mixtures $v$ due to Eq. (13), is represented by red squares, and other eigenvalues are represented by black dots.

is to characterize the class of problems that can be studied by an adiabatic tuning of the exchange coefficients $J_{k}$ in Hamiltonians of the form $\mathcal{H}$ [Eq. (1)].

\section{HOMOGENEOUS CASE: CONNECTION TO THE BETHE ANSATZ}

\section{A. Explicit eigenvalues}

We now consider the homogeneous case where $J_{1}=\cdots=$ $J_{N}=1$. In this situation the spectrum of the path graph $P_{N}$ and cycle graph $C_{N}$ are well known and are, respectively, given by [33]

$$
\sigma\left(P_{N}\right)=\left\{2-2 \cos \left(\frac{j \pi}{N}\right), 0 \leqslant j \leqslant N-1\right\}
$$

and

$$
\sigma\left(C_{N}\right)=\left\{2-2 \cos \left(\frac{2 j \pi}{N}\right), 0 \leqslant j \leqslant N-1\right\} .
$$


In particular, Eq. (17) implies that, for any mixture $v$, we get an explicit expression for the energy gap:

$$
\lambda_{*}=2-2 \cos \left(\frac{b \pi}{N}\right),
$$

with $b=1$ in the $\mathrm{OBC}$ case and $b=2$ in the PBC case. Equation (21) has been checked for several examples, such as the ones provided in Fig. 2. We observe that the energy gap vanishes as $(b \pi / N)^{2}$ in the thermodynamic limit $N \rightarrow$ $\infty$. In terms of the aforementioned applications to adiabatic quantum computing, this means that the coupling constant $J_{k}$ should be strong [typically $J_{k}=O\left(N^{2}\right)$ ] in order to have a substantial energy gap.

\section{B. Bethe ansatz in the PBC case}

The homogeneous version of $\mathcal{H}$ [Eq. (1)] in the PBC case has been intensively studied. In fact, it is for the $S=1 / 2$ version of this model [see Eq. (5)] that Bethe introduced in 1931 his celebrated ansatz, an educated guess about the form of the eigenvectors and eigenvalues of $\Delta^{\left(N_{1}, N_{2}\right)}$ [7]. His solution has been later generalized to the $S U(\kappa)$ model by Sutherland [18], and to many other classes of models, ranging from bosons and fermions in the one-dimensional continuum [8-10] to the $X Y Z$ spin chain and two-dimensional ice-type models [13] - the so-called quantum integrable systems [16]. This suggests to us that the graphs $X\left(\mathfrak{S}_{v} \subset \mathfrak{S}_{N}, \tilde{S}_{C}\right)$ we introduced and their weighted versions, which correspond to models that are no longer integrable, have an important role in a large class of strongly correlated systems. We mention some of them in the next section.

Although it is not the purpose here to provide the details of this well-established theory, we find it useful to briefly recall Bethe's original solution to the $S=1 / 2 X X X$ spin chain, and its $S U(\kappa)$ generalization by Sutherland. This allows us to obtain results on the spectra of the unweighted graphs $X\left(\mathfrak{S}_{v} \subset \mathfrak{S}_{N}, \tilde{S}_{C}\right)$ that are, up to our knowledge, not familiar to mathematicians.

In the $S=1 / 2$ case where $v=\left(N_{1}, N_{2}\right) \equiv(N-M, M)$, any spin configuration $|\chi\rangle$ is characterized by the positions of the $M$ down-spins, and one can write

$$
|\psi\rangle=\sum_{1 \leqslant x_{1}<\cdots<x_{M} \leqslant N} a\left(x_{1}, \ldots, x_{M}\right)\left|x_{1}, \ldots, x_{M}\right\rangle,
$$

where $x_{i}$ is the position of the $i$ th spin-down. If $M=1$, the Schrödinger equation is easily solved, yielding the spectrum given in Eq. (20) with eigenvectors of the (Bloch) form $a\left(x_{1}\right)=A e^{i k_{1} x_{1}}$, where $A \in \mathbb{R}$ and $k_{1}=2 j \pi / N(0 \leqslant j \leqslant N-$ $1)$. Then, Bethe's intuition was that in the general case $M \geqslant 1$ the $a\left(x_{1}, \ldots, x_{M}\right)$ coefficients could always be written as a finite sum of exponentials that is now widely known as the Bethe ansatz:

$$
a\left(x_{1}, \ldots, x_{M}\right)=\sum_{P \in \mathfrak{S}_{M}} A_{P} e^{i\left(k_{P 1} x_{1}+\cdots+k_{P M} x_{M}\right)} .
$$

Then, one can show that if $a\left(x_{1}, \ldots, x_{M}\right)$ has this form and if the Schrödinger equation is verified one can relate $A_{P}$ for $P \in \mathfrak{S}_{M}$ with $A_{Q}$ for $Q=(P 1, \ldots, P(j+1), P j, \ldots, P M)=$ $(j, j+1) \circ P$ by a simple phase factor:

$$
A_{P}=-e^{-i \theta\left(k_{P j}, k_{P(j+1)}\right)} A_{Q},
$$

where

$$
\theta\left(k_{j}, k_{l}\right)=2 \arctan \frac{1}{2}\left(\cot \frac{k_{j}}{2}-\cot \frac{k_{l}}{2}\right) .
$$

In order to determine the $M$ momentumlike quantities $k_{1}, \ldots, k_{M}$, the next step is to apply PBC by writing $a\left(x_{1}, \ldots, x_{M}\right)=a\left(x_{2}, \ldots, x_{M}, x_{1}+N\right)$, which yields

$$
e^{i k_{j} N}=(-1)^{M} e^{-i \sum_{l=1}^{M} \theta\left(k_{j}, k_{l}\right)} \quad(1 \leqslant j \leqslant M),
$$

or equivalently

$$
\left(\frac{\Lambda_{j}+i / 2}{\Lambda_{j}-i / 2}\right)^{N}=\prod_{l=1, l \neq j}^{M} \frac{\Lambda_{j}-\Lambda_{l}+i}{\Lambda_{j}-\Lambda_{l}-i} \quad(1 \leqslant j \leqslant M),
$$

where $\Lambda_{j}=\frac{1}{2} \cot \frac{k_{j}}{2}$. The $M$ coupled nonlinear equations of the form of Eq. (27) are the celebrated Bethe ansatz equations. Then, Bethe showed that their solutions (with unequal $\Lambda_{j}$ 's) yield indeed all the eigenvectors of $\Delta^{(N-M, M)}$, with eigenvalues given by

$$
E=\sum_{j=1}^{M} \frac{1}{\Lambda_{j}^{2}+1 / 4}
$$

Note that PBC are crucial in order to have the correct number of coupled equations.

Interestingly, the generalization to the $S U(\kappa)$ case by Sutherland was performed 44 years after Bethe's original solution. The idea is to apply Bethe's ansatz successively $\kappa-1$ times: For a mixture $v=\left(N_{1}, N_{2} \ldots, N_{\kappa}\right)$, we first separate the $N_{1}$ spins of type 1 with the $M_{1}=N_{2}+\cdots+N_{\kappa}=N-N_{1}$ others, and treat the spins of type 1 as the spin-up and the $M_{1}$ others as the spin-down of the previous example. Then, we separate the $M_{1}$ remaining spins between the $N_{2}$ spins of type 2 and $M_{2}=N_{3}+\cdots+N_{\kappa}=M_{1}-N_{2}$ others, that we treat, respectively, as the spin-up and spin-down of the $S=1 / 2$ case, and repeat the procedure. This technique is known as the nested Bethe ansatz. We will not write the resulting Bethe ansatz equations here, which are more involved but the forms of which are very similar to the ones described above for $S=1 / 2$ (see Ref. [18] for more details).

Thus, the Bethe ansatz provides a method for diagonalizing the Laplacian matrix $\Delta^{v}$ of the unweighted Schreier graph $X\left(\mathfrak{S}_{v} \subset \mathfrak{S}_{N}, \tilde{S}_{C}\right)$. However, it should be noted that the nonlinear coupled equations of the form Eq. (27) are hard to solve in practice for even moderate values of $N$. However, in the thermodynamic limit where $N, N_{1}, \ldots, N_{\kappa} \rightarrow \infty$ while keeping $N_{j} / N$ constant, one can write the logarithmic version of the Bethe ansatz equations as coupled integral (Fredholm) equations, allowing one to derive some exact analytical results [16]. For instance, translating a result from Sutherland for the ground-state energy per particle in the balanced case $v=$ $(N / \kappa, \ldots, N / \kappa)$ in our graph-theoretical language, we get that the spectral radius of $\Delta^{(N / \kappa, \ldots, N / \kappa)}$ is given, in the large $N$ limit, by

$$
\rho\left(\Delta^{(N / \kappa, \ldots, N / \kappa)}\right) \simeq-\frac{2 N}{\kappa}\left[\Psi\left(\frac{1}{\kappa}\right)+\gamma\right],
$$

where $\Psi$ is Euler's digamma function and $\gamma=\Psi(1) \simeq$ 0.577215 is Euler's constant. In the large $\kappa$ limit, one also 
has

$$
\rho\left(\Delta^{(N / \kappa, \ldots, N / \kappa)}\right) \simeq 2 N \sum_{k=2}^{\infty} \frac{(-1)^{k} \zeta(k)}{\kappa^{k}},
$$

with $\zeta(k)=\sum_{n \geqslant 1} 1 / n^{k}$ the Riemann zeta function. We have checked Eq. (29) by explicitly diagonalizing $\Delta^{(N / \kappa, \ldots, N / \kappa)}$ for different values of $N$ and $\kappa$. We found that this equation is already valid with less than a $1 \%$ relative error for $N=12$.

To conclude this discussion on the Bethe ansatz, we see that analytical results obtained from the theory of quantum integrable systems can be used to study the Laplacian spectra of Schreier graphs of the permutation group $\mathfrak{S}_{N}$ for large values of $N$. The approach here may potentially interest mathematicians working in the fields of asymptotic, combinatorial, and geometric group theories.

\section{OTHER QUANTUM AND CLASSICAL EXCHANGE MODELS}

\section{A. Other models described by $X\left(\mathfrak{S}_{v} \subset \mathfrak{S}_{N}, \tilde{S}_{C}\right)$}

\section{Quantum models}

So far we have focused on the Heisenberg model and its $S U(\kappa)$ generalization. In Ref. [21], we have shown that strongly repulsive $S U(\kappa)$ mixtures of ultracold fermions confined in 1D continuous potentials can also be efficiently described using the $X\left(\mathfrak{S}_{v} \subset \mathfrak{S}_{N}, \tilde{S}_{C}\right)$ graphs. This is due to a mapping between this model and a discrete spin chain [the OBC version of $\mathcal{H}$, Eq. (1)], as initially observed in Refs. [2,3]. Subsequently, other mappings allow one to extend our graph description to other models.

A first example is the Hubbard chain, which can be mapped to the Heisenberg model using the well-known Jordan-Wigner transformation [34]. More precisely, let us consider a system consisting of $M$ spinless fermions on an $N$-site chain that is described by the following Hamiltonian:

$$
\begin{aligned}
\mathcal{H}_{\mathrm{F}}= & -t \sum_{k=1}^{\tilde{N}}\left[c_{k+1}^{\dagger} c_{k}+\text { H.c. }\right] \\
& +V \sum_{k=1}^{\tilde{N}}\left(c_{k+1}^{\dagger} c_{k+1}-\frac{1}{2}\right)\left(c_{k}^{\dagger} c_{k}-\frac{1}{2}\right),
\end{aligned}
$$

where $c_{k}^{\dagger}$ and $c_{k}$ are the fermionic creation and annihilation, respectively, at site $k$. Then, $\mathcal{H}_{\mathrm{F}}$ is equivalent to the homogeneous Heisenberg spin chain $\mathcal{H}_{X X X}$ [Eq. (5)] with $J_{1}=\cdots=J_{k}=2 t=V$. Therefore, it can be described by $X\left(\mathfrak{S}_{(N-M, M)} \subset \mathfrak{S}_{N}, \tilde{S}_{C}\right)$, and results derived in Sec. II can be applied here.

\section{Classical models: A deck of cards}

Perhaps more surprisingly, this framework can also be applied to classical physics, in the context of random walks. Indeed, the Laplacian of a graph is related to the transition matrix of a random walk on this graph [35]. Here, a random walk on $X\left(\mathfrak{S}_{\nu} \subset \mathfrak{S}_{N}, \tilde{S}_{C}\right)$ can be interpreted as a so-called interchange process [1]. For example, consider a deck of $N$ cards of types $v=\left(N_{1}, \ldots, N_{\kappa}\right)\left(N_{i}\right.$ cards of type $\left.i\right)$, placed on an open or closed chain. The number of card configurations $\chi$ is $D_{\nu}$, as defined in Eq. (7). Then, at each step, a random pair of adjacent cards is selected and exchanged with a probability $J_{k} \in[0,1]$ such that $\sum_{k=1}^{\tilde{N}} J_{k}=1$. In the uniform case, one has $J_{k}=1 / \tilde{N}$ for each $k$. The transition matrix $T^{v}$ of this Markov process, which specifies the probability of going from a configuration $\chi$ to a configuration $\chi^{\prime}$, is given by

$$
T_{\chi \chi^{\prime}}^{v}= \begin{cases}0 & \text { if } \chi \neq(k, k+1) \chi^{\prime} \\ J_{k} & \text { if } \chi=(k, k+1) \chi^{\prime} \text { and } \chi \neq \chi^{\prime}, \\ 1-d(\chi) & \text { if } \chi=\chi^{\prime}\end{cases}
$$

where $d(\chi)$ is the degree of the vertex corresponding to $\chi$ in $X\left(\mathfrak{S}_{v} \subset \mathfrak{S}_{N}, \tilde{S}_{C}\right)$. Then, it is clear that $T^{v}$ is related to the Laplacian matrix $\Delta^{v}$ of $X\left(\mathfrak{S}_{v} \subset \mathfrak{S}_{N}, \tilde{S}_{C}\right)$ by

$$
T^{v}=\mathbb{1}-\Delta_{\nu} .
$$

In particular, the eigenvalues $1=\beta_{0}>\beta_{1} \geqslant \cdots \geqslant \beta_{N-1}>$ -1 of $T^{v}$ are trivially related to the spectrum of $\Delta^{v}$. As it is well known in probability theory, the spectrum of a transition matrix of a Markov process is related to its rate to stationarity, or in simpler words in our case to the speed at which a deck of cards can be considered as fully randomized by our interchange shuffle. Thus, the distance $\left\|\left(T^{v}\right)^{m}-\mu\right\|$ to the stationary measure $\mu$ after $m$ steps (see, e.g., Refs. [1,36,37] for standard definitions of the norm $\|\cdot\|$ and the stationary measure $\mu)$ is related to $\beta_{*}=\max \left(\beta_{1},\left|\beta_{N-1}\right|\right)$ by [36]

$$
\left\|\left(T^{v}\right)^{m}-\mu\right\| \leqslant \frac{\sqrt{\tilde{N}}}{2} \beta_{*}^{m} .
$$

In Sec. II, we have shown that the spectral gap of $\Delta^{v}$ in the ferromagnetic case $J_{k}>0$ is equal to the spectral gap $\lambda_{*}$ of $\Delta(N-1,1)$ for every mixture $v$. Therefore, Eqs. (33) and (34) yield $\beta_{1}=1-\lambda_{*}$. In particular in the uniform case $J_{1}=\cdots=J_{\tilde{N}}=1 / \tilde{N}$, using Eq. (21), we have found

$$
\beta_{1}=1-\frac{2}{\tilde{N}}+\frac{2}{\tilde{N}} \cos \left(\frac{b \pi}{N}\right) .
$$

Moreover, one has $\left|\beta_{N-1}\right|=\rho\left(\Delta^{v}\right)-1$, with $\rho\left(\Delta^{v}\right)$ the spectral radius of $\Delta^{\nu}$, for which there is no analytical formula in the general case. For a uniform process on a closed chain, in the balanced case $N_{1}=\cdots=N_{\kappa}=N / \kappa$ and for sufficiently large values of $N$, one may use the Bethe ansatz result of Eq. (29) and get

$$
\left|\beta_{N-1}\right| \simeq-\frac{2}{\kappa}\left[\Psi\left(\frac{1}{\kappa}\right)+\gamma\right]-1 .
$$

Additionally, one may also use one of the well-known bounds on the Laplacian spectral radii of graphs [see, e.g., Eq. (A1)]. More generally, all the results derived in the previous sections, such as Eqs. (12) and (13), or the generalized Lieb-Mattis theorem, can also be translated in this context. Thus, we see that our framework can also efficiently be used in order to analyze interchange processes.

It is important to note that in the case where the interchange process consists of $\kappa=2$ types of cards it is equivalent to the so-called symmetric exclusion process, where $M$ particles are on a chain of $N$ sites, and at each step a particle is randomly selected and jumps either to the right or to the 
(a)

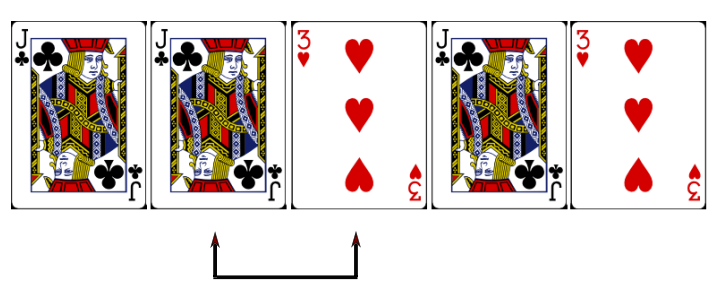

(b)

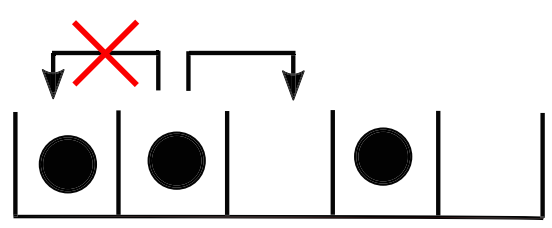

(c)

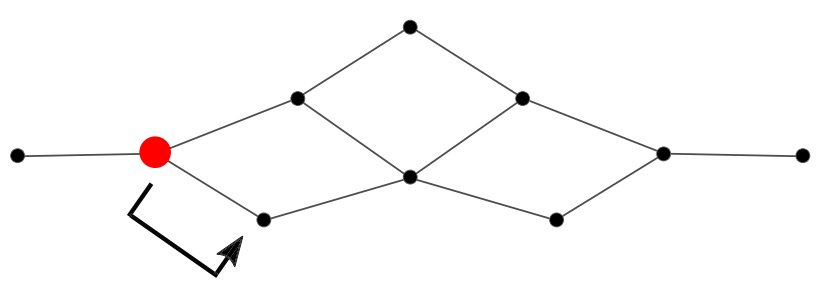

FIG. 4. Three equivalent random processes. (a) An interchange process with three black cards and two red cards. (b) A symmetric exclusion process with three particles on five sites. (c) A random walk on $X\left(\mathfrak{S}_{(3,2)} \subset \mathfrak{S}_{5}, \tilde{S}_{C}\right)$.

left if the corresponding site is empty [38]. In this case, the link to the uniform Heisenberg model and the Bethe ansatz, which is clear with our graph description, has already been exploited by mathematicians in order to study some properties of these stochastic processes (see, e.g., Ref. [37]). The equivalence between interchange and exclusion processes as well as our graph description of these models are summarized in Fig. 4.

\section{B. Generalizations}

Finally, with some ad hoc modifications, it is possible to extend our graph-theoretical description to other models. We now proceed to briefly describe two examples of such models.

The first example is the so-called asymmetric exclusion processes. As its name suggests, it is the same as symmetric exclusion processes, except that the probability $p_{k}$ of a particle on site $k$ to jump on an empty site on the left is different from the probability $q_{k}$ of jumping to the right [Fig. 5(a)]. Such models are paradigmatic in the context of nonequilibrium statistical physics, and describe a wide range of phenomena, ranging from protein synthesis on RNA [5], hopping conductivity in solid electrolytes [39], and surface growth processes [40] to traffic flows [41] and molecular rotors [42]. Similarly to the symmetric exclusion processes, these models are soluble with the Bethe ansatz in the uniform case [15]. (a)

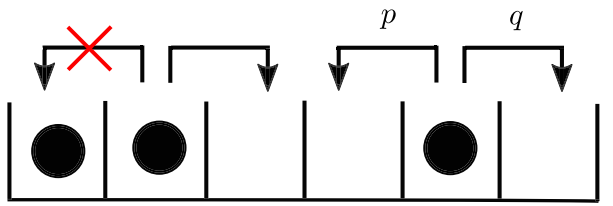

(b)

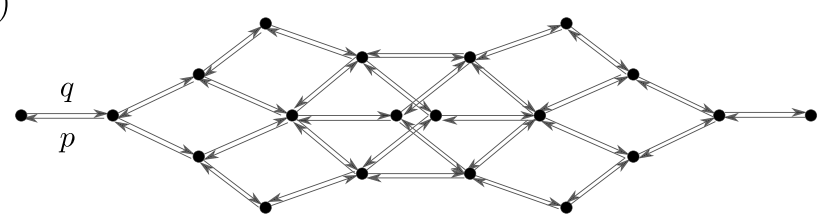

FIG. 5. (a) Illustration of an asymmetric exclusion process, with probability $p=1-q \in[0,1]$ and $p \neq q$, and, alternatively, an illustration of a non-Hermitian Hubbard model with asymmetric hopping. (b) Directed graph $X_{\rightleftarrows}\left(\mathfrak{S}_{(3,3)} \subset \mathfrak{S}_{6}, \tilde{S}_{C}\right)$ associated with panel (a). The edges corresponding to a hopping to the left or right have a weight $p$ or $q$, respectively.

Our second example is a non-Hermitian version of Eq. (31), that is, a modified Hubbard model of the form

$$
\begin{aligned}
\mathcal{H}_{\mathrm{NH}}= & -\sum_{k=1}^{\tilde{N}} t_{k}\left[e^{\alpha} c_{k+1}^{\dagger} c_{k}+e^{-\alpha} c_{k}^{\dagger} c_{k+1}\right] \\
& +\sum_{k=1}^{\tilde{N}} V_{k}\left(c_{k+1}^{\dagger} c_{k+1}-\frac{1}{2}\right)\left(c_{k}^{\dagger} c_{k}-\frac{1}{2}\right),
\end{aligned}
$$

with $\alpha>0$. Although not quantum, non-Hermitian models of this type have attracted a lot of attention recently, both theoretically and experimentally, as they effectively describe quantum systems that are coupled to their environment [43-47].

As one could suspect, those two models can both be described by the directed versions $X_{\rightleftarrows}\left(\mathfrak{S}_{(N-M, M)} \subset \mathfrak{S}_{N}, \tilde{S}_{C}\right)$ of the $X\left(\mathfrak{S}_{(N-M, M)} \subset \mathfrak{S}_{N}, \tilde{S}_{C}\right)$ graphs [see Fig. 5(b)]. Obviously, the fact that their Laplacian matrices $\Delta_{\rightleftarrows}^{v}$ are no longer symmetric makes their spectral properties more difficult to study - in particular, their spectra are not necessarily real. However, the identification of each vertex as a tabloid, and therefore the algebraic structure, is, for instance, still valid (see Sec. II). Moreover, the mapping between asymmetric exclusion processes and non-Hermitian quantum models [48] is transparent within our framework, since they are both described by the spectral properties of $\Delta_{\rightleftarrows}^{\nu}$. A more precise study of this matrix is left for future work.

\section{CONCLUSION}

In this paper, we have shown that a wide range of classical and quantum 1D exchange models are described by the same theoretical object, namely, the Laplacian matrix of a Schreier graph associated with the permutation group. This unifying description allows one to identify the algebraic structure of the problem in a natural way. As a consequence, one may access some peculiar eigenvalues much more easily, such as the spectral radius and the spectral gap, which are associated with the rate to stationarity in the classical stochastic case, and with the speed at which an adiabatic protocol may be performed 
in the context of adiabatic quantum computing. In particular, our result on the energy gap of inhomogeneous ferromagnetic Heisenberg spin chains may be tested in experiments, and has important potential applications for quantum technologies.

Furthermore, we stressed that the graphs we defined in this paper are deeply related to the celebrated Bethe ansatz, which can be regarded as a powerful tool in order to compute their spectra in the unweighted case. As an illustration, we obtained the spectral radii of Schreier graphs associated with the permutation group with a large number of elements. More generally, we believe these graphs have a central importance in the theory of quantum integrable systems. Nevertheless, we lay emphasis on the fact that our description goes beyond the integrable case, as it also enables one to describe completely inhomogeneous systems.

There are many open questions on these mathematical objects, which once tackled would give crucial information about all the models we described above. Moreover, an interesting and ambitious perspective for future works, besides the ones mentioned in this paper, would be to extend this framework to integer spin chains. Then, the fact that our method is efficient in order to study the energy gap suggests to us that it could be well adapted to tackle the celebrated Haldane conjecture on antiferromagnetic integer spin chains [49,50].

In conclusion, this paper stands at the crossroad between quantum physics, classical physics, and pure mathematics. This connection sheds light on some well-known problems, and paves the way to fruitful collaborations between different research fields.

\section{ACKNOWLEDGMENTS}

This work has been partially funded by National Research Foundation (NRF) Singapore, under Grants No.NRFNRFI2017-04 (WBS Grant No. R-144-000-378-281) and No. NRF-NRFF2018-02.

$$
\left(\begin{array}{ccc}
J_{2} & -J_{2} & 0 \\
-J_{2} & J_{1}+J_{2}+J_{3} & -J_{3} \\
0 & -J_{3} & J_{1}+J_{3} \\
0 & -J_{1} & 0 \\
0 & 0 & -J_{1} \\
0 & 0 & 0
\end{array}\right.
$$$$
\left.\begin{array}{ccc}
0 & 0 & 0 \\
-J_{1} & 0 & 0 \\
0 & -J_{1} & 0 \\
J_{1}+J_{3} & -J_{3} & 0 \\
-J_{3} & J_{1}+J_{2}+J_{3} & -J_{2} \\
0 & -J_{2} & J_{2}
\end{array}\right)
$$

and

$$
\left(\begin{array}{ccc}
J_{2}+J_{4} & -J_{2} & 0 \\
-J_{2} & J_{1}+J_{2}+J_{3}+J_{4} & -J_{3} \\
0 & -J_{3} & J_{1}+J_{3} \\
0 & -J_{1} & 0 \\
-J_{4} & 0 & -J_{1} \\
0 & -J_{4} & 0
\end{array}\right.
$$

Up to a minus sign, $\Delta_{\mathcal{G}}$ can be seen as a discrete version of the continuous Laplacian. Moreover, it is closely linked to the stochastic properties of the graph, as it can be related to the transition matrix of a random walk on $\mathcal{V}$ [35].

In the case of an undirected graph, $\mathcal{G}, \Delta_{\mathcal{G}}$ is symmetric: Thus, it can be diagonalized in an orthogonal basis and its

$$
\left.\begin{array}{c}
0 \\
-J_{4} \\
0 \\
0 \\
-J_{2} \\
J_{2}+J_{4}
\end{array}\right)
$$

\section{APPENDIX A: NOTIONS OF GRAPH THEORY}

In this section, we recall some basic definitions and properties of graph theory [51]. A graph $\mathcal{G}=(\mathcal{V}, \mathcal{E})$ is defined by a set of vertices $\mathcal{V}$ and a set of edges $\mathcal{E}$ that are characterized by a pair of vertices. When $(i, j) \in \mathcal{E}$ if and only if $(j, i) \in \mathcal{E}$, $\mathcal{G}$ is said to be undirected. A graph is weighted when each edge $e$ is associated with a real number $w_{e}$ such that all $w_{e}$ are not necessary equal, and unweighted when $w_{e}=1$ for all edges $e$. The degree $\operatorname{deg}(v)$ of a vertex $v$ is then the sum of the weights of all the edges between $v$ and its adjacent vertices. When all the vertices of a graph have the same degree $r$, the graph is said to be $r$ regular. A bipartite graph is a graph such that its vertices can be divided into two disjoint sets $\mathcal{V}_{1}$ and $\mathcal{V}_{2}$ where each edge connects an element of $\mathcal{V}_{1}$ with an element of $\mathcal{V}_{2}$. A graph $\mathcal{G}_{1}=\left(\mathcal{V}_{1}, \mathcal{E}_{1}\right)$ is a covering graph of $\mathcal{G}_{2}=\left(\mathcal{V}_{2}, \mathcal{E}_{2}\right)$ if we can map $\mathcal{G}_{2}$ into $\mathcal{G}_{1}$ while respecting the topology of the graph, i.e., if there is a surjective map $f: \mathcal{V}_{1} \rightarrow \mathcal{V}_{2}$ such that, for each $v \in \mathcal{V}_{1}$, the restriction of $f$ to a neighborhood of $v$ is a bijection onto a neighborhood of $f(v)$.

The main branch of graph theory is arguably the so-called spectral graph theory, which consists in associating matrices to the graphs of interest and then relating the properties of the graph to the spectra of their matrices [24]. The simplest matrix that one can associate with a graph $\mathcal{G}$ is its adjacency matrix $A_{\mathcal{G}}$, which is written in the basis of the vertices such that entry $A_{i j}$ is equal to $w_{(i, j)}$ if vertex " $i$ " is adjacent to vertex " $j$ " and zero otherwise. Another simple matrix that can be written in the same basis is the degree matrix $D_{\mathcal{G}}$, which is a diagonal matrix the diagonal entries of which are given by the degree of the corresponding vertex. Then, the Laplacian matrix is defined by $\Delta_{\mathcal{G}}=D_{\mathcal{G}}-A_{\mathcal{G}}$. For instance, one can check that the Laplacian matrices of the graphs in Figs. 1(a) and 1 (b) are, respectively, given by

spectrum $\sigma\left(\Delta_{\mathcal{G}}\right)$ is real. From the positivity of the diagonal entries of $\Delta_{\mathcal{G}}$ and the fact that each of them is equal to the sum of the absolute values of the nondiagonal entries in their respective row, one can easily deduce that $\sigma\left(\Delta_{\mathcal{G}}\right)$ is also positive. Since every row sum is equal to zero, the vector $u=(1,1, \ldots, 1)$ always satisfies $\Delta_{\mathcal{G}} u=0$, and we 
see that $\sigma\left(\Delta_{\mathcal{G}}\right)$ always contains zero. In fact, it is easy to show that the multiplicity of zero is equal to the number of connected components of $\mathcal{G}$. Therefore, we see that the second smallest eigenvalue $\lambda_{*}$, or spectral gap, is closely related to the topology of $\mathcal{G}$, which explains why this quantity has been intensively studied by mathematicians [52,53]. Intuitively, $\lambda_{*}$ can be seen as a measure of the connectivity of $\mathcal{G}$. Another quantity related to the geometrical properties of the graph is the spectral radius $\rho\left(\Delta_{\mathcal{G}}\right)$, which in our case is equal to the largest eigenvalue of $\Delta_{\mathcal{G}}$. One important property in the case of a connected graph is, e.g., [54]

$$
\rho\left(\Delta_{\mathcal{G}}\right) \leqslant \max \{\operatorname{deg}(u)+\operatorname{deg}(v) ;(u, v) \in \mathcal{E}\},
$$

with equality if $\mathcal{G}$ is bipartite and regular.

\section{APPENDIX B: REPRESENTATIONS OF $\mathfrak{S}_{N}$}

A complete description of the set IR of irreducible representation (irreps) of the permutation group $\mathfrak{S}_{N}$ can be found in Ref. [30].

The set IR is in bijection with the conjugacy classes of $\mathfrak{S}_{N}$ (two elements $g_{1}$ and $g_{2}$ of a group are conjugate if there is an element $h$ in the group such that $g_{1}=h^{-1} g_{2} h$ ), which are uniquely labeled by the different structures of their decomposition in disjoint cyclic permutations. Therefore, there is a one-to-one correspondence between IR and the set of partitions of $N$. Equivalently, each partition $\mu=\left[\mu_{1}, \ldots, \mu_{r}\right]$ of $N$ can be graphically represented by a Young diagram, which is a left-justified set of boxes with $r$ rows such that each row contains $\mu_{i}$ boxes. For example, one of the irreps of $\mathfrak{S}_{9}$ is characterized by the partition $[5,3,1]$, or equivalently by the following Young diagram:

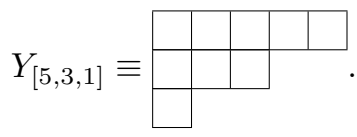

$[5,3,1]$ is also called the shape of $Y_{[5,3,1]}$. The conjugate of a Young diagram of shape $\mu=\left[\mu_{1}, \ldots, \mu_{r}\right]$ is the diagram with columns of lengths $\mu_{1}, \ldots, \mu_{r}$. For example, the conjugate of the previous example is

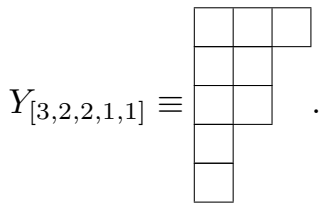

The so-called dominance order $\unrhd$ on Young diagrams is such that $\mu \equiv\left[\mu_{1}, \ldots, \mu_{r}\right] \unrhd v \equiv\left[v_{1}, \ldots, v_{r}\right]$ (where the last terms of one of the partitions may be equal to zero) if

$$
\mu_{1}+\cdots+\mu_{k} \geqslant v_{1}+\cdots+v_{k} \text { for all } 1 \leqslant k \leqslant r .
$$

For example, one has

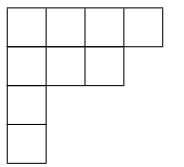

$\underline{\square}$

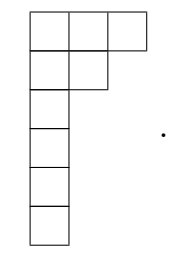

In more visual terms, it means that one can go from the left diagram to the right one by moving a certain number of boxes from upper rows to lower rows and right to left. Note that the dominance order is only a partial order when $N>5$. For instance, it is not possible to compare

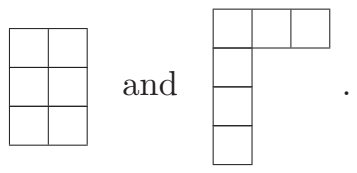

Given a Young diagram, one may associate different Young tableaux by labeling its boxes by integers. A tableau is said to be standard when its entries are increasing from left to right along the rows and up to down along the columns.

Two tableaux of same shape $\mu$ are said to be row equivalent if they are equal up to permutations of integers belonging to the same rows. For example,

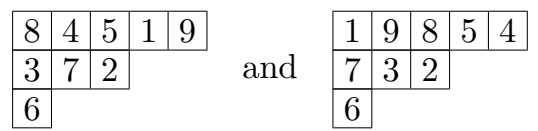

are row equivalent. Then, we define tabloids as the rowequivalence classes among the set of $\mu$ tableaux. For the pre-

\begin{tabular}{|c|c|c|c|c|}
\hline 1 & 4 & 5 & 8 & 9 \\
\hline 2 & 3 & 7 & & \\
\hline
\end{tabular}
vious example, the corresponding tabloid may be graphically represented as

Note that the number of $\mu$ tabloids is $D_{\mu}$, as defined in Eq. (7). The permutation module is then defined as the $\mathbb{C}$-vector space $M^{\mu}$ the basis of which is indexed by the set of $\mu$ tabloids. This vector space is crucial for the construction of IR. As an important peculiar case, $M^{[1,1, \ldots, 1]}$ is equivalent to $\mathbb{C}\left[\mathfrak{S}_{N}\right]$, the group algebra of $\mathfrak{S}_{N}$, the basis $\left(e_{P}\right)_{P \in \mathfrak{S}_{N}}$ of which is indexed by the elements of $\mathfrak{S}_{N}$. Additionally, the internal multiplication on $\mathbb{C}\left[\mathfrak{S}_{N}\right]$ verifies $e_{P} e_{Q}=e_{P Q}$. Then, one can naturally associate the regular representation $\mathcal{R}$ with $\mathbb{C}\left[\mathfrak{S}_{N}\right]$ by considering the canonical action of $\mathfrak{S}_{N}$ on $\mathbb{C}\left[\mathfrak{S}_{N}\right]$ : Writing a vector in $\mathbb{C}\left[\mathfrak{S}_{N}\right]$ as $u=\sum_{Q \in \mathfrak{S}_{N}} u_{Q} e_{Q}$ with $u_{Q} \in \mathbb{C}$, and given $P \in \mathfrak{S}_{N}$, the linear map $\mathcal{R}(P)$ is defined by

$$
\mathcal{R}(P) u=e_{P} u=\sum_{Q \in \mathfrak{S}_{N}} u_{Q} e_{P Q}=\sum_{Q \in \mathfrak{S}_{N}} u_{P^{-1}} e_{Q},
$$

which can be linearly extended on a representation of $\mathbb{C}\left[\mathfrak{S}_{N}\right]$ by writing $\mathcal{R}\left(\sum_{Q \in \mathfrak{S}_{N}} u_{Q} e_{Q}\right) \equiv \sum_{Q \in \mathfrak{S}_{N}} u_{Q} \mathcal{R}(Q)$. Similarly, one can identify any permutation module $M^{\mu}$ with a representation of $\mathfrak{S}_{N}$ by considering the natural action of $\mathfrak{S}_{N}$ on the vector space $M^{\mu}$.

Given a $\mu$ tableau $T$, one may associate the following element of $M^{\mu}$ known as a polytabloid:

$$
E_{T}=\sum_{P \in C_{T}} \epsilon(P)\{P(T)\},
$$

where $C_{T}$ is the set of permutations preserving all columns of $T, \epsilon(P)$ is the sign of the permutation $P$, and $\{T\}$ is the tabloid corresponding to $T$. The Specht module $S_{\mu}$ is then defined as the subspace of $M_{\mu}$ generated by all the elements $E_{T}$ when 
$T$ runs through all the $\mu$ tableaux. It can be shown that the basis of $S^{\mu}$ is given by the elements $E_{T}$ when $T$ runs though all the standard Young tableaux of shape $\mu$. In particular, its dimension is given by the number of standard Young tableaux, which is given by the so-called hook length formula. Furthermore, a crucial theorem known as Young's rule states that the permutation module $M^{\mu}$ can be decomposed in the following way:

$$
M^{\mu} \cong \bigoplus_{\mu^{\prime} \unrhd \mu} k_{\mu^{\prime} \mu} S^{\mu^{\prime}} \quad\left(k_{\mu \mu}=1\right),
$$

where $k_{\mu^{\prime} \mu}$ are positive integers called the Kostka numbers.
As it is the case for the permutation modules and the group algebra, $S^{\mu}$ can be identified with a representation of $\mathfrak{S}_{N}$ by considering the natural action of $\mathfrak{S}_{N}$ on $S^{\mu}$. Then, it can be shown that the set of all the Specht modules $S^{\mu}$ is, in fact, IR. Note that when taking $\mu=[1,1, \ldots, 1]$ Eq. (B10) implies in particular that the regular representation is a sum of all the irreps. In this case, the $k_{\mu^{\prime}[1,1, \ldots, 1]}$ numbers are given by the dimensions of the $S^{\mu^{\prime}}$ irreps. Intuitively, keeping in mind Eq. (B9), an irrep $S_{\mu}$ can be seen as symmetrizing the rows and antisymmetrizing the columns of the $\mu$ tableaux [55].
[1] P. Diaconis, Group Representations in Probability and Statistics (Institute of Mathematical Statistics, Hayward, 1988).

[2] A. G. Volosniev, D. V. Fedorov, A. S. Jensen, N. T. Zinner, and M. Valiente, Strongly interacting confined quantum systems in one dimension, Nat. Commun. 5, 5300 (2014).

[3] F. Deuretzbacher, D. Becker, J. Bjerlin, S. M. Reimann, and L. Santos, Quantum magnetism without lattices in strongly interacting one-dimensional spinor gases, Phys. Rev. A 90, 013611 (2014).

[4] W. Heisenberg, Zur theorie des ferromagnetismus, Z. Phys. 49, 619 (1928).

[5] C. T. MacDonald and J. H. Gibbs, Concerning the kinetics of polypeptide synthesis on polyribosomes, Biopolymers 7, 707 (1969).

[6] D. C. Mattis, The Theory of Magnetism (Springer-Verlag, Berlin, 1981), Vol. 1.

[7] H. Bethe, Zur Theorie der Metalle. I. Eigenwerte und Eigenfunktionen der linearen Atomkette, Z. Phys. 71, 205 (1931).

[8] E. H. Lieb and W. Liniger, Exact analysis of an interacting bose gas. i. the general solution and the ground state, Phys. Rev. 130, 1605 (1963).

[9] C. N. Yang, Some Exact Results for the Many-Body Problem in one Dimension with Repulsive Delta-Function Interaction, Phys. Rev. Lett. 19, 1312 (1967).

[10] B. Sutherland, Further Results for the Many-Body Problem in One Dimension, Phys. Rev. Lett. 20, 98 (1968).

[11] N. Andrei, K. Furuya, and J. H. Lowenstein, Solution of the Kondo problem, Rev. Mod. Phys. 55, 331 (1983).

[12] J. Ambjørn, R. A. Janik, and C. Kristjansen, Wrapping interactions and a new source of corrections to the spin-chain/string duality, Nucl. Phys. B 736, 288 (2006).

[13] R. J. Baxter, Eight-Vertex Model in Lattice Statistics, Phys. Rev. Lett. 26, 832 (1971).

[14] M. T. Batchelor, R. J. Baxter, M. J. O’Rourke, and C. M. Yung, Exact solution and interfacial tension of the six-vertex model with anti-periodic boundary conditions, J. Phys. A 28, 2759 (1995).

[15] O. Golinelli and K. Mallik, The asymmetric simple exclusion process: an integrable model for non-equilibrium statistical mechanics, J. Phys. A: Math. Gen. 39, 12679 (2006).

[16] B. Sutherland, Beautiful Models: 70 Years of Exactly Solved Quantum Many-Body Problems (World Scientific, Singapore, 2004).
[17] V. E. Korepin, N. M. Bogoliubov, and A. G. Izergin, Quantum Inverse Scattering Method and Correlation Functions (Cambridge University, Cambridge, England, 1993).

[18] B. Sutherland, Model for a multicomponent quantum system, Phys. Rev. B 12, 3795 (1975).

[19] E. Lieb and D. Mattis, Theory of ferromagnetism and the ordering of electronic energy levels, Phys. Rev. 125, 164 (1962).

[20] T. Albash and D. A. Lidar, Adiabatic quantum computation, Rev. Mod. Phys. 90, 015002 (2018).

[21] J. Decamp, J. Gong, H. Loh, and C. Miniatura, Graph-theory treatment of one-dimensional strongly repulsive fermions, Phys. Rev. Research 2, 023059 (2020).

[22] P. A. M. Dirac, The Principles of Quantum Mechanics, 4th ed. (Clarendon, Oxford, 1958).

[23] W. Pfeifer, The Lie Algebras $S U(N)$ : An Introduction (Birkhäuser, Basel, 2003).

[24] A. E. Brouwer and W. H. Haemers, Spectra of Graphs (Springer, New York, 2012).

[25] A subset $S$ of a multiplicative group $G$ is generating if one can obtain any element of $G$ by multiplying elements of $S$.

[26] W. Fulton and J. Harris, Representation Theory (Springer, New York, 2004).

[27] G. James and M. Liebeck, Representations and Characters of Groups, 2nd ed. (Cambridge University, Cambridge, England, 2001).

[28] M. Hammermesh, Group Theory and its Applications to Physical Problems (Dover, New York, 1989).

[29] For the construction of the irreducible representations of the permutation group $\mathfrak{S}_{N}$ and the definitions of the associated concepts (tabloid, permutation module, Young's rule), see Appendix B.

[30] G. James and A. Kerber, The Representation Theory of the Symmetric Group (Cambridge University, Cambridge, England, 1984).

[31] C. Poignard, T. Perreira, and J. P. Pade, Spectra of laplacian matrices of weighted graphs: Structural genericity properties, SIAM J. Appl. Math. 78, 372 (2018).

[32] R. Bacher, Valeur propre minimale du laplacien de Coxeter pour le groupe symétrique, J. Albegra 167, 460 (1994).

[33] W. N. Anderson and T. D. Morley, Eigenvalues of the Laplacian of a graph, Linear Multilinear A 18, 141 (1985).

[34] P. Jordan and E. Wigner, Über das Paulische Äquivalensverbot, Z. Phys. 47, 631 (1928). 
[35] L. Lovásk, Random Walks on Graphs: A Survey (János Bolyai Math. Soc., Budapest, 1996).

[36] P. Diaconis and L. Saloff-Coste, Comparison theorems for reversible Markov chains, Ann. Appl. Prob. 3, 696 (1993).

[37] L. L. Ng, Heisenberg Model, Bethe Ansatz, and Random Walks (Senior Honor Thesis, Harvard University, 1996).

[38] Notice that the classical equivalence between interchange and exclusion processes is analogous to the mapping between quantum Heisenberg spin-chains and the Hubbard model via the Jordan-Wigner transformation.

[39] P. M. Richards, Theory of one-dimensional hopping conductivity and diffusion, Phys. Rev. B 16, 1393 (1977).

[40] J. Krug, Origins of scale invariance in growth processes, Adv. Phys. 46, 139 (1997).

[41] M. Schreckenberg and D. E. Wolf, Traffic and Granular Flow '97 (Springer-Verlag, New-York, 1998).

[42] S. Klumpp and R. Lipowsky, Traffic of molecular motors through tube-like compartments, J. Stat. Phys. 113, 233 (2003).

[43] M. Nakagawa, N. Kawakami, and M. Ueda, Non-Hermitian Kondo Effect in Ultracold Alkaline-Earth Atoms, Phys. Rev. Lett. 121, 203001 (2018).

[44] H. Zhao, X. Qiao, T. Wu, B. Midya, S. Longhi, and L. Feng, Non-hermitian topological light steering, Science 365, 1163 (2019).

[45] Z. Yang and J. Hu, Non-hermitian hopf-link exceptional line semimetals, Phys. Rev. B 99, 081102 (2019).
[46] F. Song, S. Yao, and Z. Wang, Non-Hermitian Skin Effect and Chiral Damping in Open Quantum Systems, Phys. Rev. Lett. 123, 170401 (2019).

[47] S. Mu, C. H. Lee, L. Li, and J. Gong, Emergent Fermi surface in a many-body non-Hermitian Fermionic chain, arXiv:1911.00023 (2019).

[48] L.-H. Gwa and H. Spohn, Bethe solution for the dynamical exponent of the noisy Burger equation, Phys. Rev. A 46, 844 (1992).

[49] F. D. M. Haldane, Nonlinear Field Theory of Large-Spin Heisenberg Antiferromagnets: Semiclassically Quantized Solitons of the One-Dimensional Easy-Axis Néel State, Phys. Rev. Lett. 50, 1153 (1983).

[50] F. D. M. Haldane, Continuum dynamics of the 1-D Heisenberg antiferromagnet: Identification with the $\mathrm{O}(3)$ nonlinear sigma model, Phys. Lett. A 93, 464 (1983).

[51] J. Bondy and U. Murty, Graph Theory (Springer-Verlag, London, 2008).

[52] F. Chung, Spectral Graph Theory (American Mathematical Society, 1996).

[53] C. Godsil and G. Royle, Algebric Graph Theory (Springer, New York, 2001).

[54] B. Mohar, The Laplacian Spectrum of Graphs (Wiley, 1991).

[55] As it is typically done, we use (quite abusively) the same notation for the permutation/Specht modules and the corresponding representations. 